\title{
GENERAL DESCRIPTION OF THE SĂLAJ FLORA
}

\author{
Gavril NEGREAN ${ }^{1}$, Carol KARÁCSONYI ${ }^{2}$, Paul-Marian SZATMARI ${ }^{3,4}$ \\ ${ }^{1} 55$ Iuliu Maniu Bd., RO-77531 Bucureşti, Romania \\ ${ }^{2}$ Allgäustr.2, D-88212- Ravensburg, Germany \\ ${ }^{3}$ Biological Research Center, Botanical Garden "Vasile Fati” Jibou, \\ 16 Wesselényi Miklós St., RO-455200 Jibou, Romania \\ ${ }^{4}$ Babeș-Bolyai University, 42 Republicii St., RO-400015 Cluj-Napoca, Romania \\ email: paul_marian87s@yahoo.com
}

\begin{abstract}
In this paper we present a general description of the flora of Sălaj County. The work is based on literature data and especially on our field research. The inventory consists of more than 2000 species and subspecies, and the results are remarkably interesting. The number of taxa is much higher than we expected. This is due to the rather varied landforms, except real mountains (more than 1000 a.s.l.), but mainly because of the presence of the extensive areas covered by limestone and gypsum. The region is situated at the junction of some major geographical areas: the Transylvanian Plain in the east, the Pannonian Plain in the west, the Eastern Carpathians in the north, and the Apuseni Mountains in the south.

A number of genera were identified as new for this region (Arnica, Blechnum, Cynoglottis, Klasea, Menyanthes, Neslia, Ophioglossum, Oxytropis, Parnassia, Puccinellia, Scopolia, Waldsteinia, etc.). Also, some rare species for Romania are particularly important from a phytogeographical point of view (Anagallis minima, Aphanes arvensis, Arabis recta, Artemisia alba, Cardamine parviflora, Myosotis discolor, Rumex thyrsiflorus, Sorbus danubialis, etc.). Many species new to Romania, important from a sozological point of view, were discovered. Some of these are of national interest, others are endangered at a European level. In the area 44 endemic and subendemic species have been found (Carpathian, Dacian, Pannonian, etc.): Aconitum moldavicum, Centaurea scabiosa subsp. sadleriana, Cirsium furiens, Helleborus purpurascens, Phyteuma tetramerum, Thlaspi jankae, Viola jooi, etc. The most important are the Transylvanian Basin endemics: Jurinea transylvanica, Onosma pseudoarenaria, Salvia transsylvanica, Cephalaria radiata. There are 16 Species of Community Interest (e.g. Cypripedium calceolus, Eleocharis carniolica, Galanthus nivalis, Huperzia selago, Lycopodium clavatum, Sphagnum spp.), 13 species from the Romanian Red Book (e.g. Ophrys apifera, Sagina subulata, Gypsophila collina), and 124 taxa are included in the National Red Lists of Romania (e.g. Cephalanthera spp., Echinops ritro subsp. ruthenicus, Dactylorhiza spp., Daphne cneorum, Dictamnus albus, Erucastrum nasturtiifolium, Neottia ovata, Neotinea tridentata, Orchis purpurea).
\end{abstract}

Keywords: Sălaj flora, general description, taxonomy, sozology, Romania, Transylvania and Crişana regions

\section{Introduction}

At first glance, the flora of the Sălaj region was less known with respect to surrounding areas (Maramureş, Satu-Mare, Bihor, Cluj). This is the reason why we have decided to carry out research, first Negrean and Karácsonyi, and after a while with our younger colleague Szatmari. However, the bibliographical research has shown that there are some works regarding the flora of this territory, but these are very dispersed and many have been lost in obscurity. There are about 900 papers, which in one way or another are related to botany.

The geographical landscape of Sălaj County is rather hilly, although it is surpassed by 
two 'mountains' of quite large surface area. They do not exceed an altitude of $1000 \mathrm{~m}$. The rest of the landscape is formed by hills, crossed by several important waters: Someș in the northeast, Crasna and Barcău in the west, Agrij and Almaș in the middle, building the Agrij-Almaș Depression. There are no real plains, only smaller or larger riversides which follow the main rivers. From the floristic point of view, the calcareous and gypsum areas are the most important. They impart a clear thermophilous character to the flora, and it is perhaps because of their presence that we have an unexpectedly large number of plants present here. The limestones comprise more important landforms in the north-eastern part (Prisnel Ridge) and in the central part of the Meseș Mountains (Buciumi area), where large surfaces are covered by gypsum and calcareous formations. The most important gypsum areas are encountered in the southern part of the county, near the border with Cluj County.

\section{The history of botanical research in the Sălaj region}

Our researches, scattered at the beginning, started in the 1970s at Şimleul Silvaniei (Karácsonyi, 10.IV.1971, 1.IX.1977), Sărmăşag (Negrean, 1.IX.1977), Şimleul Silvaniei (Negrean, 2.IX.1977) and Bizuşa-Băi (Negrean, 3.IX.1998). Without knowing each other, by extraordinary coincidence these two botanists were at the same place with a difference of just one day (Şimleul Silvaniei - Karácsonyi, 1.IX.1977; Negrean 2.IX.1977). Beginning with the year 2012 the research was carried out systematically.

The oldest botanical information comes from ethnobotany, a field that still maintains names of plants very little explored [1, 40]. Many medieval acts, revealed by Petri [58], show numerous toponyms based on plant names. For example, in the oldest document from 1349, is mentioned the Willow at Dioşad (Dioasad), and in the year 1400 the oak grove at Zalău. In the recent years, there are multiple published works regarding these ethnic toponyms [7, 26, 77, 78]; even a dictionary regarding this field has been published [6]. It seems that the name "Sălaj" derives from “szil”, which means in Hungarian „elm” (Ulmus) - Szilágy (Sălaj) [33]. According to other sources, the name comes from the Latin "silva, silvae", which means "forest". In fact, in those times, the area was mostly covered by large forests [33].

The oldest information regarding the flora were given by Baumgarten [3], who, in the year 1813, at the invitation of Count Cserey Farkas (1773-1842), visited Crasna, where Cserey possessed a botanical garden. According to some information, about 1000 plant species were cultivated here [33]. On that occasion, Baumgarten visited the surroundings of Crasna village and included some species from there in his work regarding the flora of Transylvania, a first document of this kind in the country [3]. The important guest honored Cserey with an eponym: Silene csereii Baumg., a new species for science, discovered in the surroundings of Deva city.

Between 1871 and 1873, Josef Freyn (1845-1903), an engineer from Prague and a great amateur botanist, worked on the Budapest-Braşov railway. He collected a series of plant species from the southern region of Sălaj, where the railway is passing through, especially from the surroundings of Stana railway station. These plants were published by Vincentio de Borbás [25]. Also, in those times, the botanist and doctor Feichtinger Sándor crossed the western part of the actual Sălaj region, called then Crasna. He published a great number of plants, among which are many rarities [23].

Erasmus Julius Nyárády (1881-1966) carried out some research over a few months at Meseșenii de Jos, where he collected many plants, some being very interesting. These were 
included in the collection of the „Haáz Rezső” Museum at Odorheiul Secuiesc, and they have been published recently [68].

After 1940, the flora of the region began to be more actively researched. In August 1940, the former professor of Gavril Negrean from the Natural Sciences „Vasile Lucaciu” High School in Carei city, collected plants on the Puguior Hill near Ortelec village [62]. Then, in 1941, Balázs Ferenc (1913-2012) carried out serious phytocoenological research in the Meseș Mountains. In his phytocoenological tables he published around 1000 plants, and separately another 300 [2]. At the same time, another phytocoenological paper was published by Ujvárosi $\mathrm{M}$. from the surroundings of Stana village [76]. In the 1940s Tamássy Géza published some plants from the surroundings of Şimleul Silvaniei $[69,70]$. In the 1950s, the region was perambulated by Iuliu Prodan, who published plants in different articles and in the Romanian Flora [64], for example, a species with the only known locality in Romania, Carex vulpinoidea Michx., "invasive in the orchards of the village Cehu-Silvaniei" [61]. After 1960, many researchers published phytocoenological papers, especially Gheorghe Coldea [9, 10, 11, 12, 13, 15]. His doctoral thesis regarding the flora and the vegetation of Plopiș Mountains comprises a part from the Sălaj region. After this, Coldea and his collaborators continued the research [14, 16]. Other botanists have studied the vegetation in different parts of the region [37, 28, 31]. The gypsum areas from the southern limit of the county were studied by Szabó [66] and Pop [60], and other authors carried out research in the entire ethnographical area of the Călata region [53]. More recently, Molnár and his collaborators made studies in this area [44].

The interfluve of Agrij-Almăj was researched mainly by the botanists from the Agronomic University in Cluj-Napoca: Antal Nyárády and his collaborators [50]. Data from the Sălaj region are also included in many papers regarding the chorology of some species in Romania [18, 45, 63, 71].

There are several pieces of doctoral research regarding entirely [42] or partially [10, 32, 59] the flora of Sălaj, even very good graduate papers [40, 43]. Other botanists collected seeds for botanic gardens, published in seed catalogues.

Other descriptions of the Sălaj flora were recently carried out by the authors $[47,35,48]$.

\section{Evolution of the vegetation in the region}

Several papers discuss the evolution of the vegetation in this area. Paleobotanists have made numerous surveys regarding the Tertiary flora $[19,20,41,49,54,55,56,57]$. Also, Feurdean \& al. [24] and Grindean \& al. [27] carried out pollen analysis for the Quaternary vegetation. Bitiri \& Carciumaru showed that around 20.000 years ago, in a full glacial period (Würm), coniferous forests existed here. They identified Abies, Larix and Picea pollen in the north-eastern part of Sălaj County, which means that the vegetation from the Carpathian Mountains had descended very low into the lowland areas to find shelter from the extreme cold $[4]$.

As is known, the current landscape configuration is the result of the evolution of the landforms over time. The flora has also evolved, not only because of the changes in the relief, but also in response to the gradual cooling of the climate toward the end of the Tertiary. For example, the endemic Carpathian lilac species Syringa josikaea has some isolated populations, encountered in the Apuseni Mountains and in the Eastern Carpathians of Ukraine. In 2013, a team of biologists assembled some maps with the potential distribution area of the species, based 
on climatic niche analyses, for the Last Glacial Maximum [39]. Thus, we can speculate that there may have existed a paleopopulation that stretched from the Apuseni Mts to the Upper Tisa Basin towards the north via Agrij Valley, throughout Sălaj County. Molecular evidence suggests that there are no clear genetic differences between the two major populations; thus the species had a much wider range than at the present day [38].

\section{Materials and Methods}

The field researches were carried out between 2012 and 2017. We made some observations and plant lists, and the critical plants were collected to analyze them in the laboratory. They were identified according to Romanian and European taxonomic keys: [64, 75, $74,8,65,36,72,79]$. The plants were deposited in public herbaria (Carei, Cluj, Jibou, Bucharest, Galati, Iasi, Budapest, etc.). Herbarium abbreviations follow Holmgren \& al. [29] and Thiers [73].

\section{Results and Discussions}

Over five years of research, more than 2000 major taxa have been identified. This represents almost half of the Romanian flora. Among these taxa, over 1000 species are new for the Sălaj region. There are several new genera (Arnica, Blechnum, Menyanthes, Ophioglossum, Ophrys, Oxytropis, Parnassia, Puccinellia, Scopolia, Waldsteinia) or rare species in Romania (Artemisia alba, Anagallis minima, Aphanes arvensis, Arabis recta, Myosotis discolor, Rumex thyrsiflorus, Sorbus danubialis, Waldsteinia geoides etc.). From the sozological analysis we can conclude that the flora of the Sălaj region conserves species with a medium sozological character, in comparison with other areas much richer than Sălaj.

The toponyms of the plants have been published in a book that deals with the flora and the vegetation of Sălaj County [48].

We consider that the most important are the endemics. From this category in Sălaj were confirmed 44 species (Table 1.). One of the most interesting seems to be Centaurea scabiosa subsp. sadleriana, a species that was not found in Romania for over 50 years. The population is represented by very few individuals, so the species needs an urgent conservation programme.

In this category we have the Transylvanian endemics, followed by the Dacian, Carpathian and Pannonian species. In recent papers the majority of the Dacian species are considered to be also Carpathian elements [30].

Regarding Natura 2000 species, in Sălaj were identified 16 taxa of vascular plants and bryophytes: 11 vascular plants (3 Lycopodiaceae) and 6 Bryophytes. Most of them have a single population in the county and are critically endangered. The most frequent is Galanthus nivalis [80] (Table 2).

In the county there are 13 species from the Red Book of the Romanian Flora. Among these is Gypsophila collina, which forms important populations especially on the gypsum between Sfăraş and Jebucu. This species is at the westernmost edge of its distribution area [22] (Table 3). 
Table 1: Endemic and subendemic species in Sălaj County

\begin{tabular}{|c|c|c|}
\hline 1. & Aconitum moldavicum & Carpathian element \\
\hline 2. & Cardamine glanduligera & Sub-Carpathian element \\
\hline 3. & $\begin{array}{l}\text { Centaurea jacea subsp. banatica (syn. } \\
\text { Centaurea rocheliana) }\end{array}$ & Daco-Pannonic-(N) Balkan element \\
\hline 4. & Centaurea phrygia subsp. indurata & Daco-Balkan-Pannonic element \\
\hline 5. & Centaurea pugioniformis & Daco-Transylvanian element \\
\hline 6. & Centaurea scabiosa subsp. sadleriana & Pannonic element \\
\hline 7. & Cephalaria radiata & Transylvanian element \\
\hline 8. & Cirsium furiens & Pannonic-Dacian element \\
\hline 9. & Crocus banaticus & Sub-Carpathian element \\
\hline 10. & Crocus vernus subsp. vernus & Alpine (E)-Balkan (NW)-Carpathian element \\
\hline 11. & Dactylorhiza maculata subsp. transsilvanica & Daco-(NW) Balkan element \\
\hline 12. & Delphinium simonkaianum & Carpathian element \\
\hline 13. & Galium abaujense & Carpathian element \\
\hline 14. & Galium eruptivum & Daco-Pannonic element \\
\hline 15. & Helictotrichon decorum & Carpathian element \\
\hline 16. & Helleborus purpurascens & Carpatho-Pannonic-(N) Balkan-Dacian element \\
\hline 17. & Jurinea transylvanica & Transylvanian element \\
\hline 18. & Melampyrum barbatum & Apennin-Pannonic-Balkan-Dacian element \\
\hline 19. & Onosma pseudoarenaria & Transylvanian element \\
\hline 20. & Phyteuma tetramerum & Carpathian element \\
\hline 21. & Pulsatilla montana subsp. dacica & Transylvanian element \\
\hline 22. & Rhinanthus wagneri & Carpatho-(N) Balkan element \\
\hline 23. & Rosa bohemica var. negreanii & local endemic element \\
\hline 24. & Rosa pocsii var. karacsonyii & local endemic element \\
\hline 25. & Rosa pocsii var. pocsii & local endemic element \\
\hline 26. & Rubus cîrlioarae & local endemic element \\
\hline 27. & Rubus $\times$ jibouensis & local endemic element \\
\hline 28. & Rubus $\times$ silasensis & local endemic element \\
\hline 29. & Rubus subvillicaulis & local endemic element \\
\hline 30. & Rubus $\times$ tunelensis & local endemic element \\
\hline 31. & Rubus vaccarum & local endemic element \\
\hline 32. & Salvia transsylvanica & Transylvanian element \\
\hline 33. & Scabiosa columbaria subsp. banatica & Carpathian element \\
\hline 34. & Scabiosa columbaria subsp. pseudobanatica & Carpathian element \\
\hline 35. & Seseli gracile & Daco-(NW) Balkan element \\
\hline 36. & Sesleria heufleriana subsp. heufleriana & Carpathian element \\
\hline 37. & Silene heuffelii & Carpatho-Balcanic element \\
\hline 38. & Silene nutans subsp. dubia & Carpathian element \\
\hline 39. & Silene otites subsp. hungarica & Apennin-Carpatho-(NW) Balkan element \\
\hline 40. & Symphytum cordatum & Carpathian element \\
\hline 41. & Thlaspi jankae & Carpatho-Pannonic element \\
\hline 42. & Thymus comosus & Carpathian element \\
\hline 43. & Thymus dacicus & Daco-(NW) Balkan element \\
\hline 44. & Viola jooi & Carpathian element \\
\hline
\end{tabular}


Table 2: Community interest species (Natura 2000 species)

\begin{tabular}{|c|c|c|c|}
\hline & Species & Observation 1 & Observation 2 \\
\hline 1 & Adenophora liliifolia & [44: 117] & 1 choronim! \\
\hline 2 & Arnica montana & & 2 choronims \\
\hline 3 & Cypripedium calceolus & $\begin{array}{l}\text { In the southern part of Gălăşeni is a } \\
\text { large population with } 49 \text { flowering } \\
\text { individuals. }\end{array}$ & 4-5 choronims \\
\hline 4 & Pontechium maculatum & $\begin{array}{l}\text { Syn. Echium maculatum, E. rubrum, } \\
\text { E. russicum }\end{array}$ & 1 choronim! (Guruslău) \\
\hline 5 & Eleocharis carniolica & $\begin{array}{l}\text { Very few individuals, usually on the } \\
\text { edges of small ponds }\end{array}$ & 3 choronims \\
\hline 6 & Galanthus nivalis & Usually in beech forests & The most frequent \\
\hline 7 & Huperzia selago & Populations with few individuals & 5 choronims \\
\hline 8 & Iris aphylla & Populations with few individuals & 3 choronims \\
\hline 9 & Leucobryum glaucum & & 3 choronims \\
\hline 10 & Lycopodium clavatum & Sometimes frequent & 10 choronims \\
\hline 11 & $\begin{array}{l}\text { Lycopodium } \\
\text { complanatum }\end{array}$ & Very rare & 1 choronim! \\
\hline 12 & Sphagnum flexuosum & $\begin{array}{l}\text { Syn. S. amblyphyllum: The Swamp at } \\
\text { Iaz }\end{array}$ & 1 choronim! \\
\hline 13 & Sphagnum capillifolium & & 1 choronim! \\
\hline 14 & Sphagnum contortum & $\begin{array}{l}\text { Published by Balázs } 1942 \text { as } \\
\text { Sphagnum riparium, revised by } \\
\text { Boros }[2,48]\end{array}$ & 1 choronim! \\
\hline 15 & Sphagnum subsecundum & $\begin{array}{l}\text { Published by Balázs } 1942 \text { as } \\
\text { Sphagnum riparium, revised by } \\
\text { Boros }[2,48]\end{array}$ & 2 choronims \\
\hline 16 & $\begin{array}{l}\text { Sphagnum } \\
\text { magellanicum }\end{array}$ & Only in the Swamp at Iaz & 1 choronim! \\
\hline
\end{tabular}

Table 3: Rare plants from the Red Book of Romania present in Sălaj County

\begin{tabular}{l|l|l}
\hline $\mathbf{1}$ & Alyssum montanum subsp. montanum & $\begin{array}{l}\text { Meseş Mts., Ortelec, Mirșid \& Sângeorgiul de Meseş - } \\
\text { Buciumi }\end{array}$ \\
\hline $\mathbf{2}$ & Carex supina & 1 choronim [Herbarium sheet CLA 23.860] \\
\hline $\mathbf{3}$ & Centaurea scabiosa subsp. sadleriana & 1 choronim (Carastelec), C. Karácsonyi \\
\hline $\mathbf{4}$ & Gypsophila collina & $\begin{array}{l}\text { Was treated under G. fastigiata; only between Sfăraş and } \\
\text { Jebucu, and also at Gălăşeni limestone quarry }\end{array}$ \\
\hline $\mathbf{5}$ & Lysimachia nemorum & Treznea, 29 VI 2015, G. Negrean [Herbarium BUC CL]. \\
\hline $\mathbf{6}$ & Myosotis discolor & 3 choronims \\
\hline $\mathbf{7}$ & Onosma pseudoarenaria & Populations with a few individuals; Endangered! \\
\hline $\mathbf{8}$ & Ophrys apifera & 4 choronims [67] \\
\hline $\mathbf{9}$ & Rhinanthus alectorolophus & Plopiș Mts., 31 V 2016, G. Negrean [Herbarium BUC]. \\
\hline $\mathbf{1 0}$ & Rumex thyrsiflorus & More than 10 choronims \\
\hline $\mathbf{1 1}$ & Sagina subulata & Very rare; only 6 choronims \\
\hline $\mathbf{1 2}$ & Thlaspi jankae & Riseg Hill; stable population \\
\hline $\mathbf{1 3}$ & $\begin{array}{l}\text { Tripidium strictum (syn. Saccharum } \\
\text { strictum) }\end{array}$ & $\begin{array}{l}\text { Meseşenii de Jos, 3 VIII 1903, E. I. Nyárády [ODORHEI } \\
7115 / 137] \text { [68: 65] }\end{array}$ \\
\hline
\end{tabular}

In Sălaj County we also identified a total of 124 plants from the main four Red Lists regarding the Romanian Flora (Table 4). From these, 27 are qualified as Vulnerable, species that 
should be included in a protected area. Also, there are 26 orchid species that are in general protected at a European level, and other species from the flora of Romania that are very rare in this region: Aconitum variegatum, Aira elegantissima, Astragalus austriacus, Asyneuma canescens, Epipactis greuterii, Equisetum fluviatile, Helictotrichon compressum, Iris pumila, Iris ruthenica, Leontodon saxatilis, Scopolia carniolica, Sedum album, Seseli elatum subsp. osseum, Silene gallica, Sonchus palustris, Viola alba subsp. scotophylla, etc. More than 200 species are rare plants.

Table 4: Species from the main National Red Lists (some of them were not refound*; the symbol ${ }^{\triangleleft}$ represent the synonyms of the species in different publications)

\begin{tabular}{|c|c|c|c|c|c|}
\hline No. & Taxa & O \& al. & B\&al. & D\&D & GN \\
\hline 1. & Achillea ptarmica & - & - & - & $\mathrm{R}$ \\
\hline 2. & Adenophora liliifolia & - & - & $\mathrm{V}$ & $\mathrm{V}$ \\
\hline 3. & Adonis vernalis & - & - & - & $\mathrm{V}$ \\
\hline 4. & Agrostemma githago & - & $\mathrm{V}$ & - & - \\
\hline 5. & Amygdalus nana & $\mathrm{V}$ & - & - & - \\
\hline 6. & Anacamptis coriophora (syn. Orchis coriophora ${ }^{\star}$ ) & $\mathrm{R}^{4}$ & - & - & $\mathrm{R}^{4}$ \\
\hline 7. & Anacamptis morio subsp. morio & $\mathrm{R}^{4}$ & - & - & $\mathrm{R}^{4}$ \\
\hline 8. & Anacamptis morio subsp. picta & $\mathrm{R}^{4}$ & - & - & $\mathrm{R}^{\top}$ \\
\hline 9. & $\begin{array}{l}\text { Anacamptis palustris subsp. elegans (syn. Orchis palustris } \\
\text { subsp. elegans, O. laxiflora subsp. elegans } \ \text { ) }\end{array}$ & $\mathrm{R}^{4}$ & - & - & $\mathrm{R}^{4}$ \\
\hline 10. & Arnica montana & V & - & - & $\mathrm{R}$ \\
\hline 11. & Artemisia alba & $\mathrm{R}$ & $\mathrm{R}$ & $\mathrm{R}$ & $\mathrm{R}$ \\
\hline 12. & Bupleurum rotundifolium & - & V & - & - \\
\hline 13. & Calamagrostis canescens & - & - & $\mathrm{R}$ & $\mathrm{R}$ \\
\hline 14. & Cardamine parviflora & $\mathrm{R}$ & - & V & $\mathrm{R}$ \\
\hline 15. & Carex diandra & $\mathrm{R}$ & - & $\mathrm{R}$ & $\mathrm{R}$ \\
\hline 16. & Carex limosa & $\mathrm{R}$ & - & $\mathrm{R}$ & $\mathrm{R}$ \\
\hline 17. & Carex secalina & $\mathrm{R}$ & - & $\mathrm{R}(\mathrm{V})$ & $\mathrm{R}$ \\
\hline 18. & Carlina acanthifolia subsp. utzka & $\mathrm{R}^{*}$ & - & $\mathrm{R}$ & $\mathrm{R}$ \\
\hline 19. & Centaurea calocephala (syn. C. atropurpurea) & $\mathrm{R}^{4}$ & - & $\mathrm{R}^{4}$ & $\mathrm{R}^{4}$ \\
\hline 20. & Centaurea scabiosa subsp. sadleriana & $\mathrm{R}$ & $\mathrm{R}$ & E & $\mathrm{R}$ \\
\hline 21. & Cephalanthera damasonium & $\mathrm{R}$ & - & - & - \\
\hline 22. & Cephalanthera longifolia & $\mathrm{nt}$ & - & - & - \\
\hline 23. & Cephalanthera rubra & $\mathrm{R}$ & - & $\mathrm{R}$ & - \\
\hline 24. & Cephalaria radiate & $\mathrm{R}$ & - & $\mathrm{R}$ & $\mathrm{R}$ \\
\hline 25. & Cerastium gracile & - & - & $\mathrm{R}$ & $\mathrm{R}$ \\
\hline 26. & Cirsium eriophorum & $\mathrm{K}^{*}$ & - & - & $\mathrm{R}$ \\
\hline 27. & Cirsium furiens & $\mathrm{nt} *$ & - & $\mathrm{R}$ & $\mathrm{nt}$ \\
\hline 28. & Crepis pannonica & $\mathrm{K}$ & - & - & $\mathrm{E}$ \\
\hline 29. & Crocus banaticus & - & - & - & $\mathrm{V} / \mathrm{R}$ \\
\hline 30. & Cypripedium calceolus & $\mathrm{V} / \mathrm{R}$ & $\mathrm{E}$ & $\mathrm{V}$ & $\mathrm{V} / \mathrm{R}$ \\
\hline 31. & Dactylorhiza incarnata & $\mathrm{R}$ & - & - & $\mathrm{R}$ \\
\hline 32. & Dactylorhiza maculata s. 1 & $\mathrm{R}$ & - & - & $\mathrm{R}$ \\
\hline 33. & Dactylorhiza maculata subsp. transsilvanica & $\mathrm{R}$ & - & - & $\mathrm{R}$ \\
\hline 34. & Dactylorhiza sambucina subsp. sambucina & $\mathrm{R}$ & - & - & $\mathrm{R}$ \\
\hline 35. & Daphne cneorum & $\mathrm{V} / \mathrm{R}$ & $\mathrm{V}$ & $\mathrm{V}$ & $\mathrm{V}$ \\
\hline 36. & Delphinium fissum & $\mathrm{R}$ & - & - & $\mathrm{R}$ \\
\hline 37. & Delphinium simonkaianum & $\mathrm{R}$ & - & $\mathrm{V} / \mathrm{R}$ & $\mathrm{R}$ \\
\hline
\end{tabular}




\begin{tabular}{|c|c|c|c|c|c|}
\hline 38. & Dictamnus albus & $\mathrm{V} / \mathrm{R}$ & - & - & - \\
\hline 39. & Drosera rotundifolia & $\mathrm{R}$ & - & - & $\mathrm{R}$ \\
\hline 40. & Echinops ritro subsp. ruthenicus & $\mathrm{R}$ & - & - & $\mathrm{R}$ \\
\hline 41. & Eleocharis carniolica & - & - & $\mathrm{V}$ & - \\
\hline 42. & Elymus hispidus subsp. barbulatus & $\mathrm{R}$ & - & - & - \\
\hline 43. & Epipactis helleborine & $\mathrm{R}$ & - & - & - \\
\hline 44. & Epipactis microphylla & $\mathrm{R}$ & - & $\mathrm{R}$ & - \\
\hline 45. & Epipactis palustris & - & - & - & $\mathrm{R}$ \\
\hline 46. & Epipactis purpurata & $\mathrm{R}$ & - & - & - \\
\hline 47. & Eriophorum gracile* & $\mathrm{R}$ & - & $\mathrm{R}$ & $\mathrm{R}$ \\
\hline 48. & Erucastrum nasturtiifolium subsp. nasturtiifolium & $\mathrm{R}$ & - & I & - \\
\hline 49. & Fritillaria meleagris subsp. meleagris & $\mathrm{R}$ & $\mathrm{V}$ & $\mathrm{V}$ & $\mathrm{V}$ \\
\hline 50. & Galanthus nivalis & $\mathrm{nt}$ & - & - & - \\
\hline 51. & Galium abaujense & - & - & $\mathrm{K}$ & - \\
\hline 52. & Galium parisiense & $\mathrm{R}$ & - & $\mathrm{R}$ & - \\
\hline 53. & Galium pumilum & $\mathrm{R}$ & - & $\mathrm{R}$ & $\mathrm{R}$ \\
\hline 54. & Genista germanica & $\mathrm{R}$ & - & $\mathrm{R}$ & $\mathrm{R}$ \\
\hline 55. & Gentiana pneumonanthe & - & - & - & $\mathrm{V}$ \\
\hline 56. & Geranium purpureum & $\mathrm{R}$ & - & $\mathrm{R}$ & $\mathrm{R}$ \\
\hline 57. & Gladiolus imbricatus & - & - & $\mathrm{R}$ & - \\
\hline 58. & Gymnadenia conopsea & $\mathrm{R}$ & - & - & - \\
\hline 59. & Gypsophila collina & $\mathrm{R}$ & $\mathrm{I}$ & $\mathrm{K}$ & - \\
\hline 60. & Hottonia palustris & $\mathrm{V} / \mathrm{R}^{4}$ & $\mathrm{~V}$ & $\mathrm{~V}$ & - \\
\hline 61. & Hyoscyamus niger & - & $\mathrm{R}$ & - & - \\
\hline 62. & Iris aphylla & - & - & $\mathrm{R}$ & $\mathrm{R}$ \\
\hline 63. & Iris sibirica & - & $\mathrm{V}$ & $\mathrm{R}$ & $\mathrm{R}$ \\
\hline 64. & $\begin{array}{l}\text { Jurinea transylvanica (syn. J. mollis subsp. } \\
\text { transsilvanica } 4 \text { ) }\end{array}$ & $\mathrm{R}$ & - & $\mathrm{R}$ & $\mathrm{R}^{4}$ \\
\hline 65. & Klasea radiata (syn. Serratula radiata & $\mathrm{R}^{4}$ & - & - & $\mathrm{R}^{4}$ \\
\hline 66. & Lathyrus pannonicus subsp. pannonicus & $\mathrm{R}$ & - & $\mathrm{R}$ & $\mathrm{R}$ \\
\hline 67. & Leucojum aestivum subsp. aestivum & $\mathrm{V} / \mathrm{R}$ & - & $\mathrm{V}$ & $\mathrm{V}$ \\
\hline 68. & Leucojum vernum subsp. vernum & - & - & $\mathrm{R}$ & $\mathrm{R}$ \\
\hline 69. & Listera ovata (syn. Neottia ovata) & $\mathrm{R}$ & - & - & - \\
\hline 70. & $\begin{array}{l}\text { Lycopodium complanatum (syn. Diphasium } \\
\text { complanatum } \triangleleft \text { ) }\end{array}$ & $\mathrm{R}^{4}$ & $\mathrm{R}$ & - & $\mathrm{R}$ \\
\hline 71. & Lysimachia nemorum & $\mathrm{R}$ & - & $\mathrm{R}$ & - \\
\hline 72. & Medicago prostrate & Ex & - & $\mathrm{R}(\mathrm{I})$ & $\mathrm{V}$ \\
\hline 73. & Melampyrum nemorosum & - & - & $\mathrm{R}(\mathrm{K})$ & $\mathrm{R}$ \\
\hline 74. & Menyanthes trifoliata & $\mathrm{R}$ & - & $\mathrm{R}$ & - \\
\hline 75. & Moenchia mantica [50: 81] & $\mathrm{R}$ & - & - & $\mathrm{R}$ \\
\hline 76. & Monotropa hypopytis & $\mathrm{R}$ & - & - & - \\
\hline 77. & Montia fontana & $\mathrm{R}$ & - & $\mathrm{E}$ & - \\
\hline 78. & Myosotis discolor & $\mathrm{R}$ & - & $\mathrm{R}$ & $\mathrm{R}$ \\
\hline 79. & $\begin{array}{l}\text { Narcissus poeticus subsp. radiiflorus (syn. Narcissus } \\
\text { stellaris } ₫ \text { ) }\end{array}$ & - & $\mathrm{V}^{4}$ & $\mathrm{~V}$ & $\mathrm{R}^{4}$ \\
\hline 80. & Neotinea tridentata (syn. Orchis tridentata $\mathbf{v}$ ) & $\mathrm{R}^{4}$ & - & - & $\mathrm{R}$ \\
\hline 81. & Neotinea ustulata (syn. Orchis ustulata) & $\mathrm{R}^{4}$ & - & - & $\mathrm{R}^{4}$ \\
\hline 82. & Onosma pseudoarenaria & $\mathrm{R}$ & - & $\mathrm{E}$ & $\mathrm{R}$ \\
\hline 83. & Ophrys apifera & $\mathrm{R}$ & - & $\mathrm{E}$ & $\mathrm{E}$ \\
\hline 84. & Orchis militaris & $\mathrm{R}$ & - & - & $\mathrm{R}$ \\
\hline 85. & Orchis purpurea subsp. purpurea & $\mathrm{R}$ & - & - & $\mathrm{R}$ \\
\hline
\end{tabular}




\begin{tabular}{|c|c|c|c|c|c|}
\hline 86. & Papaver lecoquii (syn. Papaver dubium subsp. lecoquii*) & - & - & $\mathrm{V}$ & - \\
\hline 87. & Peucedanum officinale & $\mathrm{R}$ & $\mathrm{V}$ & - & $\mathrm{R}$ \\
\hline 88. & Phleum hirsutum & $\mathrm{R}$ & - & $\mathrm{K}$ & $\mathrm{R}$ \\
\hline 89. & Phyteuma tetramerum & $\mathrm{R}$ & - & - & $\mathrm{R}$ \\
\hline 90. & Piptatherum virescens & $\mathrm{R}$ & - & - & - \\
\hline 91. & Platanthera bifolia & $\mathrm{R}$ & - & - & - \\
\hline 92. & Platanthera chlorantha & $\mathrm{R}$ & - & - & - \\
\hline 93. & Rhinanthus borbasii subsp. borbasii & $\mathrm{R}$ & - & $\mathrm{R}$ & $\mathrm{R}$ \\
\hline 94. & Rhinanthus wagneri & $\mathrm{R}$ & - & - & $\mathrm{R}$ \\
\hline 95. & Rorippa islandica & $\mathrm{R}$ & - & - & - \\
\hline 96. & Rosa micrantha & $\mathrm{R}$ & - & - & - \\
\hline 97. & Rosa subcollina & - & - & $\mathrm{R}$ & - \\
\hline 98. & Rosa vosagiaca & - & $\mathrm{R}$ & - & - \\
\hline 99. & Rumex aquaticus & $\mathrm{R}$ & - & $\mathrm{R}$ & $\mathrm{R}$ \\
\hline 100. & Rumex longifolius & $\mathrm{R}$ & - & $\mathrm{R}$ & $\mathrm{R}$ \\
\hline 101. & Rumex thyrsiflorus subsp. thyrsiflorus & $\mathrm{R}$ & $\mathrm{R}$ & $\mathrm{I}$ & $\mathrm{R}$ \\
\hline 102. & Sagina subulata & $\mathrm{R}$ & $\mathrm{R}$ & $\mathrm{R}$ & $\mathrm{R}$ \\
\hline 103. & Salix aurita & $\mathrm{R}$ & - & - & - \\
\hline 104. & Salvia nutans & - & - & V & - \\
\hline 105. & Saxifraga bulbifera & $\mathrm{R}$ & V & - & $\mathrm{R}$ \\
\hline 106. & Scabiosa columbaria subsp. pseudobanatica & $\mathrm{R}$ & - & - & - \\
\hline 107. & Scorzonera austriaca & - & - & $\mathrm{V}$ & $\mathrm{R}$ \\
\hline 108. & Sedum cepaea & $\mathrm{R}$ & - & $\mathrm{R}$ & $\mathrm{R}$ \\
\hline 109. & Sempervivum marmoreum & $\mathrm{R}$ & - & - & - \\
\hline 110. & Spergula pentandra & $\mathrm{R}$ & $\mathrm{I}$ & $\mathrm{R}$ & $\mathrm{R}$ \\
\hline 111. & Spiranthes spiralis & $\mathrm{R}$ & - & - & $\mathrm{R}$ \\
\hline 112. & Stipa dasyphylla & $\mathrm{R}$ & $\mathrm{R}$ & $\mathrm{V}$ & $\mathrm{R}$ \\
\hline 113. & Stratiotes aloides & $\mathrm{V}$ & - & - & - \\
\hline 114. & Succisella inflexa & $\mathrm{V} / \mathrm{R}$ & - & $\mathrm{V}$ & $\mathrm{R}$ \\
\hline 115. & Thymus comosus & nt & - & $\mathrm{nt}$ & $\mathrm{nt}$ \\
\hline 116. & Thymus longicaulis & $\mathrm{R}$ & - & $\mathrm{R}$ & $\mathrm{R}$ \\
\hline 117. & Trapa natans & $\mathrm{V}$ & - & - & - \\
\hline 118. & Traunstenera globosa & $\mathrm{R}$ & - & - & $\mathrm{R}$ \\
\hline 119. & Trinia kitaibelii (syn. Trinia ramosissima ${ }^{\triangleleft}$ ) & - & $\mathrm{R}^{4}$ & - & - \\
\hline 120. & Tripidium strictum (syn. Saccharum strictum ${ }^{\triangleleft}$ ) & $\mathrm{R}$ & $\mathrm{R}^{4}$ & $\mathrm{E}^{4}$ & $\mathrm{R}^{4}$ \\
\hline 121. & Trollius europaeus subsp. europaeus & $\mathrm{R}$ & - & $\mathrm{V}$ & $\mathrm{R}$ \\
\hline 122. & Typha laxmannii & - & $\mathrm{R}$ & - & - \\
\hline 123. & Viola jooi & $\mathrm{R}$ & - & $\mathrm{R}$ & $\mathrm{R}$ \\
\hline 124. & Waldsteinia geoides & $\mathrm{R}$ & - & $\mathrm{R}$ & - \\
\hline
\end{tabular}

Legend: O\&al. = Oltean, Negrean \& al. 1994 [52]; B\&al. = Boşcaiu, Horeanu \& Coldea 1994 [5]; D\&D = Dihoru G. \& Dihoru A. 1994 [21]; GN = G. Negrean 2001 [46].

A total of 20 taxa were described from Sălaj, especially species from the Rosaceae family (Crataegus, Rosa and Rubus), very polymorphic species (Table 5).

During these botanical researches, we made an important new conclusion, namely: the main migration route westward of many continental (steppe) species passed through Sălaj, especially via the Ortelec Valley, on the saddle in the Buciumi area of the Meseş Mountains and very likely through the corridor of Crișul Repede River. Presumably only a few species have passed through the long and narrow corridor of the Mureș River. A description regarding this 
phenomenon, with significant maps, was presented by the authors abroad and received with great interest [34].

Table 5: Taxa described from the Sălaj region

\begin{tabular}{l}
\hline Alyssum repens Baumg. subsp. transsilvanicum (Schur) Baumgartner forma latifolia Pteancu [62] \\
\hline Crataegus lindmanii Hrab.-Uhr. subsp. kovacsii Kerényi-Nagy [48] \\
\hline Crataegus $\times$ monostevenii Pénzes ex Kerényi-Nagy nothosubsp. negreanii Kerényi-Nagy [48] \\
\hline Crataegus $\times$ subsphaerica Gand. nothosubsp. karacsonyii Kerényi-Nagy [48] \\
\hline Crataegus $\times$ subsphaerica Gand. nothosubsp. negreanii Kerényi-Nagy [48] \\
\hline Ranunculus ulmeniorum Prod. - Plant that was described by Prodan from the Sălaj region; the only \\
known choronim in the world; unknown plant! [61] \\
\hline Rosa bohemica Heinr. Braun var. negreanii Kerényi-Nagy 2013 [48] \\
\hline Rosa $\times$ geczii Kerényi-Nagy nothospec. nova 2013 [48] \\
\hline Rosa $\times$ geczii Kerényi-Nagy nm. divekyii Kerényi-Nagy \& Karácsonyi (nothomorpha nova) [48] \\
\hline Rosa pocsii Kerényi-Nagy var. pocsii [48] \\
Rosa pocsii Kerényi-Nagy var. karacsonyii Kerényi-Nagy 2013 [48] \\
\hline Rubus cîrlioarae Nyár. - „In silva Păd. Cîrlioara inter pagos Jibou et Şoimuş [51: 898]. „The Forest \\
Cîrlioara between Jibou and Soimus, 350 m” [51: 356, 359]
\end{tabular}

Rubus $\times$ jibouensis Nyár. (Rubus drautensis $\times$ thyrsanthus) - „In the Vacii Valley at Someș-Odorhei, $250 \mathrm{~m}$ (district Jibou)”. „In valle V. Vacii prope pagum Someş-Odorhei (r. Jibou)” [51: 563, 934]

Rubus $\times$ silasensis Nyár. (Rubus procerus $\times$ questieri) - „Stîna, near the tunnel and in the Silaş Forest” [51: 572]. „Ad viam ferream et in silva Silaş prope stationem Stîna” [51: 936]. Observation: The correct name of the locality is Stana, not Stîna (Zalău district)!

Rubus $\times$ strugensis Sprib. var. impeditus Nyár. (Rubus caesius $\times$ vulgaris var. stinensis) - , ad stationem viae ferreae Stîna” [51: 933]. „Stîna near the C. F. R. tunnel” [51: 559]. Observation: The correct name of the locality is Stana, not Stîna (Zalău district)!

Rubus subvillicaulis Nyár. - „Stîna on the Silaș Hill” [51: 400]

Rubus subvillicaulis Nyár. var. quinnatus Nyár. - „in monte Silaș prope stationem viae ferreae Stîna” [51: 906]. Observation: The correct name of the locality is Stana, not Stîna (Zalău district)!

Rubus $\times$ tunelensis Nyár. (Rubus suberectus $\times$ thyrsanthus) - „ad marginem silvae juxta viam prope stationem Stîna” [51: 937]. „Stîna, near the tunnel, alongside the field road” [51: 579]. Observation: The correct name of the locality is Stana, not Stîna (Zalău district)!

Rubus vaccarum Nyár. - „In the Vacii Valley at Someş-Odorhei, 250 m (district Jibou)” [51: 424, 427]. „In valle V. Vacii prope pagum Someş-Odorhei (r. Jibou)” [51: 911, 912]

Rubus vulgaris Weihe \& Nees var. stanensis Nyár. - „Stîna prope tunellum viae ferreae” [51: 898]. „Stîna near the C. F. R. tunnel” [51: 371]. N.B. The correct name of the locality is Stana, not Stîna (Zalău district)!

\section{Conclusions}

During research carried out between 2012 and 2017, more than 2000 species and subspecies have been identified in Sălaj County, with more than 20 new genera for the regional flora. A high number of endemic and subendemic species were identified in this county, the most important being those with a small distribution area, such the Transylvanian, Dacian, Pannonic and Carpathian elements. These species confer a specific character to the region.

Of the species identified, 16 are Natura 2000 taxa, and 13 are included in the Red Book of Romania. These species need protection and conservation. Also, after analyzing the flora, we found that a high number of species belong to the National Red Lists: 124 species and subspecies, of which 27 are Vulnerable. These should be included within protected areas.

20 taxa were described from Sălaj (locus classicus), mostly very polymorphic species belonging to the genera Crataegus, Rosa and Rubus. 
Also, we observed that many thermophilous species have their northern limit in this region: Aira elegantissima, Alcea pallida, Artemisia alba, Asyneuma canescens, Melampyrum barbatum, Ophrys apifera, Piptatherum virescens, Plantago argentea, Quercus frainetto, Quercus pubescens, Rhinanthus wagneri, Saxifraga bulbifera, Sedum cepaea, Seseli gracile, Smyrnium perfoliatum, Sorbus danubialis, Thlaspi alliaceum, Tilia tomentosa, etc. This fact is very important from a phytogeographical point of view. Other species are at their global western limit (Gypsophila collina), or their eastern limit (Centaurea scabiosa subsp. sadleriana, Galium abaujense, Sagina subulata) in this area.

Other species have found here a refugium during the glacial periods, and managed to survive in small refugia across the county. Some descended probably from the Gutin-Ţibleş Mountains in the north, others from the Vlădeasa Massif in the south.

We have also observed many invasive and potentially invasive species that we have not discussed in this paper, but we can say that the region presents fewer invasive species populations that other areas in the country. The majority of them are problematic mostly on the Someș riversides.

During these botanical researches, we achieved an important new conclusion regarding the westward migration route of the continental species. This is very important even at a European level, because many botanists have challenged the possibilities of where these species migrated to the Pannonian Basin.

\section{REFERENCES}

1. Băcescu, M.S., 1952, Material de folklor zoologic şi de anatomie populară din jurul Zalăului, Bul. Şti., Secţ. Şti. Biol., Agron., Geol. \& Geogr., Acad. Română, 4 (4): 807-831.

2. Balázs, F., 1941, Vegetációtanulmányok a Meszes hegységben, Acta Geobot. Hung., 4 (1): 119-182.

3. Baumgarten, J.C.G., 1816/1817, Enumeratio Stirpium magno Transsilvaniae Principatui praeprimis indigenarum, Vindobonae, 1 \& 2, [Vindobonae: Libreria Camesina. 1 xxvii, 427 pp., 2 (12), 392 pp., in 1816; 3, in 1817 xii, 355 pp.].

4. Bitiri, M., Carciumaru, M., 1980, Primele dovezi de cultură materială şi artă paleolitică în judeţul Sălaj, Acta Mvs. Porolissensis (Zalău), 4: 17-23.

5. Boşcaiu, N., Coldea, G., Horeanu, C., 1994, Lista Roşie a plantelor vasculare dispărute, periclitate, vulnerabile şi rare din flora României, Ocrot. Nat., 38 (1): 45-56.

6. Chende-Roman, G., 2006, Dicţionar etimologic al localităţilor din judeţul Sălaj, Editura Silvana, Editura Caiete Silvane.

7. Chişu, Ş., 1992, Sensuri şi semnificaţii în toponimia satului Şoimuş (I), Acta Mvs. Porolissensis (Zalău), 16: 585-612.

8. Ciocârlan, V., 2009, Flora ilustrată a României - Pteridophyta et Spermatophyta. /Ediţia a III-a/. Editura Ceres, Bucureşti, 1141 pp.

9. Coldea, G., 1971, Diagrama sporo-polinică a mlaştinii de la Băile Iaz (Munţii Plopiş), Stud. Cercet. Biol., ser. Bot., 23 (5): 405-408.

10. Coldea, G., 1972, Flora şi vegetaţia Munţilor Plopiş, Rezumat teză de doctorat, Univ. Babeş-Bolyai, ClujNapoca, 28 pp.

11. Coldea, G., 1979, Pajiştile de luncă din zona Munţilor Plopiş, Contrib. Bot., /ser. II/, /1979: 163-174.

12. Coldea, G., 1981, Pajiştile mezofile din Munţii Plopiş, Stud. Cercet. Biol., ser. Biol. Veg., 33 (1): 45-54.

13. Coldea, G., 1984, Vegetaţia lemnoasă de luncă din zona Munţilor Plopiş, Acta Mus. Porolissensis (Zalău), 8: 565-570. 
14. Coldea, G., Miclăuş, V., 1975, Contribuţii la studiul stejăretelor din piemontul Nord-estic al Munţilor Plopiş "Pădurea Lăpişul" (jud. Sălaj), Contr. Bot. /ser. II/, /1975: 121-127.

15. Coldea, G., Pop, A., 1994, Über die Saumgesellschaften (Trifolio-Geranietea Th. Müller 61) aus Siebenbürgen - On the Transylvanian forest-border vegetation (Trifolio-Geranietea Th. Müller 61), (Siebenbürg. Archiv 30), Naturwiss. Forsch. Siebenb, 5: 63-76.

16. Coldea, G., Täuber, F., Plămadă, E., Pop, A., Bartók, E., Munteanu, D., 1987, Cercetări biocenologice în ecosistemele forestiere din dealurile Sălajului şi Gîrboului, Acta Mus. Poroliss., Muz. Istorie şi Artă din Zalău, 11: 479-492.

17. Csergő, A.-M., 2005, A csillagos narcisz, Erdélyi Nimród Természetvédelmi Mag., 7 (3): 16-17.

18. Danciu, M., Parascan, D., Gurean, D., Ularu, P., 2000, Contribuţii la cunoaşterea răspândirii în România şi a fitocenologiei speciei Genista germanica L, Revista de Silvicultură, Anul V, 1-2 (11-12)/ 2000: 18-22.

19. Diaconeasa, B., Nicorici, E., 1964, Cercetări palinologice din depozitele Sarmaţianului inferior de la Fizeş (Bazinul Sălaj), Stud. Cercet. Geol., Geofiz., Geogr., ser. Geol., Bucureşti, 9 (2): 295-307.

20. Diaconeasa, B., Nicorici, E., 1968, Cercetări palinologice din depozitele Neogene din sudul Bazinului Şimleu. Stud. Cercet. Geol., Geofiz., Geogr., ser. Geol., Bucureşti, 13 (1): 249-256.

21. Dihoru, G., Dihoru, A., 1994, Plante rare, periclitate şi endemice în Flora României - Lista roşie • Red list of rare, threatened and endemic plants from Flora of Romania, Acta Bot. Horti Bucurest. /1993-1994/: 173-197.

22. Dihoru, G., Negrean, G., 2009, Cartea roşie a plantelor vasculare din România, Editura Academiei Române. Bucureşti, $630 \mathrm{pp}$.

23. Feichtinger, S., 1875, Krasznamegye és környéke Flórájáról, Math. Term. Tud. Közl., 1871, 9, No III: 55-115.

24. Feurdean, A., Mosbrugger, V., Onac, B.P., Polyak, V., Veres, D., 2007, Younger Dryas to mid-Holocene environmental history of the lowlands of NW Transylvania, Romania, Quaternary Research, 68 (3): 364-378.

25. Freyn, J., 1877, Az 1871-1873 évben Magyarország keleti részeiben gyüjtött növények jegyzéke, Math. Term. Közl. (1875-1876), 13: 65-130.

26. Galoş, M., 1991, Toponimia din localităţile Gălăşeni şi Tămaşa (jud. Sălaj) • The toponymy in the Gălăşeni and Tămaşa villages (Sălaj County), Acta Mus. Porolissensis (Zalău), 14-15: 607-628.

27. Grindean, R., Tanțău, I., Fărcaș, S., Panait, A., 2015, Middle to Late-Holocene vegetation shifts in the NW Transylvanian lowlands (Romania), Studia Univ. Babeș-Bolyai, Geol., 2014-2015, 59 (1-2): 29-37.

28. Hodişan, I., Şuteu, Ş., 1979, Contribuţii la cunoaşterea vegetaţiei din Cheile Babii (jud. Sălaj), Studia Univ. Babeş-Bolyai, Biol., 24 (1): 8-17.

29. Holmgren, P.K., Holmgren, N.H., Barnett. L.C., 1990, Index Herbariorum, Part I: The Herbaria of the World. 8th Ed. Regnum Veg. 120: 1-693. [Online supplements:

http://sciweb.nybg.org/science2/IndexHerbariorum.asp], http://scisun.nybg.org:8890/searchdb/owa/wwwih.search_list

30. Hurdu, B.I., Pușcaș, M., Turtureanu, P.D., Niketić, M., Vonica, G., Coldea, G., 2012, A critical evaluation of Carpathian endemic plant taxa from the Romanian Carpathians, Contrib. Bot., 47: 39-47.

31. Indreica, A., 2011, On the Occurence in Romania of Potentillo albae-Quercetum petraeae Libbert 1933 Association, Notul. Bot. Horti Agrobot. Cluj-Napoca, 39 (1): 297-306.

32. Karácsonyi, C., 2011, Flora şi vegetaţia dealurilor Tăşnadului şi a colinelor marginale, Editura „Vasile Goldiş" University Press, Arad, 368 pp.

33. Karácsonyi, K., 2012, Az ismeretlen szilágysági növényvilág, Hepehupa, Zilah (Zalah), 11 (4): 23-32.

34. Karácsonyi, K., Negrean, G., 2012, Phytogeographical characters of the West-Plains (Romania) in the light of the new floristical researches. A Romániai Nyugati-síkság növényföldrajzi jellege az újabb florisztikai kutatások tükrében. P. 29. In: 9th Recent Floristic and Vegetation Research in Carpathian Basin - International Conference, Szent István University, 24-26 $6^{\text {th }}$ February 2012. Gödöllö, Hungary. Public. in: Kitaibelia, 17 (1): 29.

35. Karácsonyi, C., Negrean, G., 2013, A szilágysági növényvilág jellegének vizsgálat, Kanitzia, Journal of Botany, 20: 101-118.

36. Király, G., (ed.), 2009, Új magyar füvészkönyv. Magyarország hajtásos növényei. Határozókulcsok, Aggteleki Nemzeti Park Igazgatóság. Jósafö. 616 pp.

37. Kovács, A., 1971, Cercetări fitocenologice în împrejurimile comunei Sărmăşag (jud. Sălaj), Studia Univ. Babeş-Bolyai, ser. Biol. /1971/ (1): 41-55. 
38. Lendvay, B., Kadereit, J.W, Westberg, E., Cornejo, C., Pedryc, A., Höhn, M., 2015, Phylogeography of Syringa josikaea (Oleaceae): Early Pleistocene divergence from East Asian relatives and survival in small populations in the Carpathians, Biological Journal of the Linnean Society.

39. Macalik, K., Tamás, R., Kolcsár, L.-P., Keresztes, L., 2013, Present status of the Syringa josikaea Jacq. ex Rchb., an endemic species which contributes to the diversity of the Flora of the Carpathians, Studia Univ. „Babeş-Bolyai”, Biol. 58 (2): 31-40.

40. Medve, M., 1990, Valorificarea florei spontane din Culmea Prisnelului, Lucrare metodico-ştiinţifică pentru obţinerea gradului didactic I. Universitatea din Cluj Napoca, Facultatea de Biologie, Geografie şi Geologie, Catedra de Botanică, 77 pp.

41. Mészáros, N., 1998, Formaţiunile terţiare din judeţul Sălaj, Natura Silvaniei (Jibou) 1/1997/: 83-96.

42. Miclăuş, V., 1968, Contribuţii la studiul solurilor din seria podzolirii argilo-iluviale, din Dealurile Silvaniei. Lucrare pentru obţinerea titlului de doctor în agronomie, Institutul Agronomic „Nicolae Bălcescu” Bucureşti, $223 \mathrm{pp}$.

43. Mihály-Sárközi, H., 1985, Flora și aspectele de vegetație din împrejurimile satului Tetișu (județul Sălaj), Lucrare de diploma, Universitatea Cluj-Napoca, Facultatea de Biologie-Geografie și Geologie, Catedra de Biologie.

44. Molnár, C., Bódisz, J., Óvári, M., Raksányi, Z., Bró, É., Gerner, G., Nagy, T., Molnár, K., Molnár, Z., 2014, Sztána és Zsobok (Kalotaszeg) flórája, Kitaibelia (Debrecen), 19 (1): 114-132.

45. Morariu, I., Danciu, M., Kovács, A., 1984, Corologia speciei Cardamine glanduligera O. Schwarz (Dentaria glandulosa W. et K.) în România, Stud. Cercet. Biol., ser. Biol. Veg., 36 (1): 35-41.

46. Negrean, G., 2001, Lista roşie a plantelor din România existente în pajişti (inclusiv endemite şi subendemite). Pp. 30-58. In: Coldea, G., Negrean, G., Sârbu, I., Sârbu, A., 2001, Ghid pentru identificarea şi inventarierea pajiştilor seminaturale din România, Bucureşti, Editura alo, 58 pp.

47. Negrean, G., Karácsonyi, C., Szatmari, P.-M., 2015, Caracterizarea generală a florei Sălajului. Pp. 18-20. In: Anonim, 2015, Sesiunea Ştiinţifică Internațională „Flora, vegetația, fauna, ecologia şi ocrotirea patrimoniului natural al Sălajului” (evaluare preliminară). Jibou, Sălaj, 13-14 noiembrie 2015. Arad: Edit. „Vasile Goldiş” Univ. Press. 47 pp.

48. Negrean, G., Karácsonyi, C., Szatmari, P.-M., 2017, Patrimoniul natural al Sălajului. Vol. 1. Flora, Micobiota și Vegetaţia • Flora, Mycobiota and the Vegetation of Sălaj County, Satu Mare: Editura Someşul, 1319 pp.

49. Nicorici, E., 1967, Studiul stratigrafic al depozitelor neogene din partea sudică a bazinului Şimleu, Teză de doctorat, Universitatea Bucureşti, Facultatea de Geologie-Geografie, 330 pp.

50. Nyárády, A., Pázmány, D., †Jula, F., Chircă, E., Szabó, A.T., 1976, Wiesen und Wälder aus dem Agrij-Almaş becken, Notul. Bot. Horti Agrobot. Cluj-Napoca, 1975/76, 8: 77-81.

51. Nyárády, E.I., 1956, Rubus L., In: Traian Săvulescu (Red.), Collaboratores Tomi IV: Beldie, A., Buia, A., Guşuleac, M., Nyárády, E.I., Prodan, I., Răvăruţ, M., Flora României • Flora Romaniae. Vol. 4. Bucureşti: Editura Academiei Române, 958 pp.

52. Oltean, M., Negrean, G., Popescu, A., Roman, N., Dihoru, G., Sanda, V., Mihăilescu, S., 1994, Lista roşie a plantelor superioare din România. In: M. Oltean (coord.), Studii, sinteze, documentatiii de ecologie, Acad. Română, Institutul de Biologie, Nr. 1: 1-52.

53. Péntek, J., Szabó, A., 1985, Ember és növényvilág. Kalotaszeg növényzete és népi növényismerete, Bucureşti: Editura Kriterion, 368 pp.

54. Petrescu, I., 1968a, Cîteva plante noi din oligocenul Văii Almaşului (jud. Sălaj), Contrib. Bot., Cluj, /ser. II/, /1968/: 403-410.

55. Petrescu, I., 1968b, Considerazioni generali sulla flora fossile della Valea Almaşului (Romania nord occidentale), Bol. Soc. Paleont. Ital., Modena, 7 (1): 78-84.

56. Petrescu, J., 1969, Flora oligocenă din bazinul Văii Almaşului (NV Transilvaniei) cu privire specială asupra celor miocen-inferioare. Vol. I-II. Teză de doctorat., Universitatea Bucureşti, Facultatea de GeologieGeografie, 275 pp.

57. Petrescu, I., Fati, V., 1997, Oscilaţii climatice la limita Oligocen - Miocen în Bazinul Almaş-Agrij (NV României) - Climatic changes at Oligocene-Miocene in the Almaş-Agrij Basin (NW Romania), Natura Silvaniei (Jibou), 1: 41-49.

58. Petri, M., 1901-1904, Szilágy vármegye monográfiája, Vol. I-VI. Budapest. 
59. Pop, A., 1985, Flora şi vegetaţia interfluviului valea Nadăşului - valea Borşei. Teză de doctorat , Univ. BabeşBolyai, Facultatea de Biologie și Geografie, Cluj-Napoca.

60. Pop, A., 1996, Floristisch-ökologische Bemerkungen zur Vegetation der Klausenburger Berge $\bullet$ Floristical and Ecological Considerations on the Vegetation of the Hills of Cluj, Stapfia, 45: 103-134.

61. Prodan, I., 1957, Contribuţii la flora R.P.R., Bul. Şti., Secț. Biol., Şti. Agric. (ser. Bot.), Acad. Română, 9 (4): 285-326.

62. Pteancu, P., 1944, O nouă formă de Alyssum transsilvanicum Schur, Bul. Grăd. Bot. Cluj, 1943, 23 (3-4): 9096.

63. Sanda, V., Negrean, G., Ştefan, N., 1992, Cartarea speciilor genului Valerianella din Flora României, Stud. Cercet. Biol., ser. Biol. Veg., 44 (2): 115-139.

64. Săvulescu, T., (ed.), 1952-1976, Flora României • Flora Romaniae. București, Editura Academiei Române. Vol. 1-13.

65. Sârbu, I., Ştefan, N., Oprea, A., 2013, Plante vasculare din România: determinator ilustrat de teren, Editura VictorBVictor, București, $1320 \mathrm{pp}$.

66. Szabó, A.T., 1983, Vegetaţia gipsofilă din sud-vestul Podişului Someşan • Gypsophilous plant communities from the south-west of the Somes plateau, Ocrot. Nat., 27 (2): 103-111.

67. Szatmari, P.-M., 2016, Ophrys apifera (Orchidaceae) in Transylvanian flora, Romania, Acta Horti Botanici Bucurestiensis, 43: 31-40.

68. Székely, V., 2007, Catalogul colecţiei de plante „Nyárády Erazmus Gyula” de la Muzeul „Haáz Rezső” din Odorheiul Secuiesc • The Catalogue of Gyula Erazmus Nyárády’s Collection of Plants, Haáz Rezső Museum, Odorheiul Secuiesc, Romania • Nyárády Erazmus Gyula növénygyüjteményének katalógusa a székelyudvarhelyi Haáz Rezső Múzeumban, Székelyudvarhely, Romania, Acta Siculica, 2007: 57-67.

69. Tamássy, G., 1942, „Apró közlemények” • Kleine Mitteilungen, A Crocus banaticus, Spiranthes spiralis s a Galinsoga aristulata lelöhelyei, Bot. Közlem., 39 (6): 639.

70. Tamássy, G., 1948, „Szilágysomlyó növényzete”, Bot. Közlem., 1947, 44: 84.

71. Täuber, F., 1974, Răspîndirea speciei Crocus banaticus Gay în Carpaţii româneşti, Tibiscus, Şti. Nat. (Timişoara), /1974/: 21-34.

72. The Plant List (2013), Version 1.1. Published on the Internet. Retrieved November 28, 2017, from: http://www.theplantlist.org/

73. Thiers, B.M., 2014, Index herbariorum a global directory of public herbaria and associated staff. New York Botanical Garden's Virtual Herbarium, URL: http://sweetgum.nybg.org./ih/

74. Tutin, T.G., Burges, N.A., Chater, A.O., Edmondson, J.R., Heywood, V.H., Moore, D.M., Valentine, D.H., Walters, S.M., Webb, D.A., 1996, Flora Europaea. 2nd ed., 1993, reprinted 1996. Vol. 1. Psilotaceae to Platanaceae. Cambridge: Cambridge University Press xlvi, 581 pp.

75. Tutin, T.G., Heywood, V.H., Burges, N.A., Moore, D.M., Valentine, D.H., Walters, S.M., Webb, D.A., (eds), 1964-1980. Flora Europaea. Vols. 1-5. Cambridge: Cambridge University Press.

76. Ujvárosi, M., 1947, Növényszociológiai vázlatok Sztána környékéröl, Borbásia, 7 (1-6): 3-27.

77. Vasiliu, G., 1984, Din toponimia satului Moigrad (I), Acta Mvs. Porolissensis (Zalău), 8: 756-761.

78. Vasiliu, G., 1985, Din toponimia satelor: Mirşid, Firminiş, Popeni, Acta Mvs. Porolissensis (Zalău), 9: 745750.

79. von Raab-Straube, E., 2017, The Euro-MedPlantbase Project/Euromed/. /acces: Euro Med, Dahlem, Berlin/.

80. *** OUG 57/2007. Ordonanţa de urgenţă nr. 57 din 20 iunie 2007 privind regimul ariilor naturale protejate, conservarea habitatelor naturale, a florei şi faunei sălbatice. Guvernul României (Natura 2000 Romanian law).

\section{DESCRIEREA GENERALĂ A FLOREI SĂLAJULUI}

\section{(Rezumat)}

În acest articol se face o descriere generală a florei Sălajului. Lucrarea se bazează pe date din literatură şi mai ales pe cercetările proprii. Lista este formată din peste 2000 de specii şi subspecii, iar rezultatele sunt surprinzător de interesante. Numărul taxonilor este cu mult mai mare față de cât ne-am fi aşteptat. Aceasta se datorează reliefului destul de variat, dar lipsit de munţi adevăraţi (de peste $1000 \mathrm{~m}$ ), situării zonei la interferenţa 
dintre unele zone geografice importante: Câmpia Transilvaniei în est, Câmpia Panonică în vest, Carpaţii Orientali în nord şi Munții Apuseni, în sud, dar mai ales prezenţei masive a calcarelor şi a gipsurilor.

Au fost identificate mai multe genuri noi pentru regiunea Sălajului (Arnica, Blechnum, Cynoglottis, Klasea, Menyanthes, Neslia, Ophioglossum, Oxytropis, Parnassia, Puccinellia, Scopolia, Waldsteinia etc.). Câteva specii rare pentru România (Anagallis minima, Aphanes arvensis, Arabis recta, Artemisia alba, Cardamine parviflora, Myosotis discolor, Rumex thyrsiflorus, Sorbus danubialis etc.) sunt deosebit de importante fitogeografic. Din punct de vedere sozologic, au fost descoperiţi mai mulţi taxoni, unii de interes naţional, alţii de interes comunitar. Au fost găsite 44 de specii endemice sau subendemice (carpatice, dacice, panonice etc.): Aconitum moldavicum, Centaurea scabiosa subsp. sadleriana, Cirsium furiens, Helleborus purpurascens, Phyteuma tetramerum, Thlaspi jankae, Viola jooi etc.). Deosebit de importante sunt endemitele Bazinului Transilvan: Jurinea transylvanica, Onosma pseudoarenaria, Salvia transsylvanica, Cephalaria radiata. Au mai fost identificate 16 specii de interes comunitar (Cypripedium calceolus, Eleocharis carniolica, Galanthus nivalis, Huperzia selago, Lycopodium clavatum, Sphagnum spp. etc.), 13 specii din Cartea Roşie: Ophrys apifera, Sagina subulata, Gypsophila collina (plantă extrem de rară la nivel European, aflându-se în Sălaj la limita de vest a arealului mondial) și 124 taxoni din listele Roşii Naţionale: Cephalanthera spp., Echinops ritro subsp. ruthenicus, Dactylorhiza spp., Daphne cneorum, Dictamnus albus, Erucastrum nasturtiifolium, Neottia ovata, Neotinea tridentata, Orchis purpurea etc. 